\title{
Knockdown of NANOG Reduces Cell Proliferation and Induces G0/G1 Cell Cycle Arrest in Human Adipose Stem Cells
}

\author{
Maria Pitrone ${ }^{1}$, Giuseppe Pizzolanti ${ }^{1,2, *(1)}$, Antonina Coppola ${ }^{1}$, Laura Tomasello ${ }^{1}$, \\ Stefania Martorana ${ }^{3}$, Gianni Pantuso ${ }^{3}$ and Carla Giordano ${ }^{1,2, *}$ \\ 1 Aldo Galluzzo Laboratory of Regenerative Medicine, Department of Health Promotion Sciences, \\ Maternal and Infant Care, Internal Medicine and Medical Specialties, PROMISE, University of Palermo, \\ 90127 Palermo, Italy; maria.pitrone@unipa.it (M.P.); antonina.coppola02@unipa.it (A.C.); \\ laura.tomasello@unipa.it (L.T.) \\ 2 ATeN (Advanced Technologies Network Center), University of Palermo, 90127 Palermo, Italy \\ 3 Department of Surgical, Oncological and Oral Sciences, Division of General and Oncological Surgery, \\ University of Palermo, 90127 Palermo, Italy; stefania.martorana@unipa.it (S.M.); \\ gianni.pantuso@unipa.it (G.P.) \\ * Correspondence: giuseppe.pizzolanti@unipa.it (G.P.); carla.giordano@unipa.it (C.G.); \\ Tel.: +39-091-6552138 (G.P.); +39-091-6552110 (C.G.); Fax: +39-091-6552138 (G.P.); +39-091-6552123 (C.G.)
}

Received: 23 April 2019; Accepted: 23 May 2019; Published: 26 May 2019

\begin{abstract}
The core components of regenerative medicine are stem cells with high self-renewal and tissue regeneration potentials. Adult stem cells can be obtained from many organs and tissues. NANOG, SOX2 and OCT4 represent the core regulatory network that suppresses differentiation-associated genes, maintaining the pluripotency of mesenchymal stem cells. The roles of NANOG in maintaining self-renewal and undifferentiated status of adult stem cells are still not perfectly established. In this study we define the effects of downregulation of NANOG in maintaining self-renewal and undifferentiated state in mesenchymal stem cells (MSCs) derived from subcutaneous adipose tissue (hASCs). hASCs were expanded and transfected in vitro with short hairpin Lentivirus targeting NANOG. Gene suppressions were achieved at both transcript and proteome levels. The effect of NANOG knockdown on proliferation after 10 passages and on the cell cycle was evaluated by proliferation assay, colony forming unit (CFU), qRT-PCR and cell cycle analysis by flow-cytometry. Moreover, NANOG involvement in differentiation ability was evaluated. We report that downregulation of NANOG revealed a decrease in the proliferation and differentiation rate, inducing cell cycle arrest by increasing $p 27 / C D K N 1 B$ (Cyclin-dependent kinase inhibitor 1B) and $p 21 / C D K N 1 A$ (Cyclin-dependent kinase inhibitor 1A) through $p 53$ and regulate DLK1/PREF1. Furthermore, NANOG induced downregulation of DNMT1, a major DNA methyltransferase responsible for maintaining methylation status during DNA replication probably involved in cell cycle regulation. Our study confirms that NANOG regulates the complex transcription network of plasticity of the cells, inducing cell cycle arrest and reducing differentiation potential.
\end{abstract}

Keywords: human adipose stem cell; NANOG; cell cycle regulation; DNMT1; lentiviral transduction

\section{Introduction}

OCT4, SOX2 and NANOG are the actors in the complex machinery that regulate pluripotency of mesenchymal stem cells [1]. Expression of OCT4 and NANOG is restricted to pluripotent cells, and they are downregulated upon differentiation [2,3]. OCT4 and NANOG work together to maintain pluripotency without LIF (Leukemia inhibitory factor) [4] and studies confirm that NANOG 
downregulation induces ESCs (Embryonic stem cell) differentiation into extraembryonic lineages [5,6]. Wang et al. showed that OCT4 and NANOG maintain self-renewal and block differentiation in ESCs [7]; however, little is known about their mechanism in adult mesenchymal stem cells. Adipose stem cells (ASCs) represent an alternative source of mesenchymal stem cells that can be easily and safely obtained from adipose tissue, growing under appropriate culture conditions for a long time [8-10]. As previously shown, ASCs express CD29, CD90, CD105 and CD73 and are negative for the hematopoietic endothelial markers CD34, CD45, CD117, and HLA-DR [11-14]. Nowadays, it is more important to understand the mechanisms through which maintain the proliferation, differentiation and heterogeneity of these cells $[15,16]$. OCT4, SOX2 and NANOG have also been suggested to play a similar role as embryonic stem cells in adult mesenchymal cells, including human Adipose Stem Cells (hASCs). In our previous studies, we found that the embryonic stem cell marker NANOG is over-expressed in MSCs derived from adipose tissue and its silencing with a RNA interference technology causes downregulation of OCT4 and SOX2 gene expression [13], confirming recent studies that have demonstrated a central role of NANOG in regulating stem cells' multipotent properties $[15,17]$.

DLK1 (PREF1) is a marker used to characterized mouse preadipocyte progenitors which inhibits adipogenesis, suppressing $C / E B P \beta$ and $C / E B P \delta$ gene expression [18-23]. Recently, DLK1 (PREF1) proved to be a useful marker for human ASCs $[24,25]$ and methylation regulates its expression. One of the DNA methyltransferase that regulates the DNA methylation during replication is the DNMT1 [25] and ESCs differentiation and embryo development are regulate by DNA methylation. A recent study [26] demonstrated that NANOG directly binds to the promoter of DNMT1 [26] and enhances its expression. In this study, our aim was to investigate the role of NANOG in maintaining the proliferation and differentiation potential of ASCs after downregulation of NANOG with a Lentivirus system and to examine the expression of some important genes related to cell growth control such as $p 27 / C D K N 1 B$, p21/CDKN1A, CCDN1 and PREF1. We show that NANOG downregulation induced a decrease in the proliferation rate and differentiation potential and led to cell cycle arrest in G0/G1 by regulating CCDN1, $p 21 / C D K N 1 A$ and $p 27 / C D K N 1 B$ through $p 53$ and a PREF1 inhibition, inducing loss of pluripotency via DNMT1.

\section{Results}

\section{1. hASC Isolation and Characterization}

Enzymatic digestion of biopsied human adipose tissue was obtained after consent from 20 patients (nine men and 11 women; age $45 \pm 10$ years; with body mass index (BMI) range $28 \pm 3$ ) undergoing elective open-abdominal surgery. As previously published, hASC highly expressed THY1, CD105 and CD73 and all of the ESC markers SOX-2, OCT4 and NANOG (see Table S1 in Supplementary). When cultured in adhesion we observed the formation of colonies of fibroblastic-like cells, whereas in low-adhesion culture conditions spheres were formed (Figure 1).

\subsection{Knockdown of NANOG Silencing by Lentivirus}

To evaluate the roles of NANOG in maintaining stem cell properties we studied changes in stem cell marker gene expression in hASCs lentiviral transducted with shRNA against NANOG. The mRNA and protein expression level of NANOG after 10 and 15 days of antibiotic selection after shRNA infection was evaluated by quantitative PCR and western blot analysis. As observed in Figure 2A, the NANOG mRNA level decreased by almost $90 \pm 3.5 \%$ in cell lines infected with NANOG shRNA lentivirus as compared to the control group (hASCs CNT, freshly isolated hASCs at passage 4, and hASCs transducted with scrambled shRNA against NANOG, hASC negative shRNA NANOG). Furthermore, Figure 2B showed the downregulation of NANOG protein expression after 15-day antibiotic selection, by almost $76 \pm 2.3 \%$ in hASCs infected with NANOG shRNA lentivirus (Optical Density $(O D)=0.87 \pm 0.9$ versus $0.2 \pm 0.06$ ). The quantitative analysis of western blot bands was performed using ImageJ. In our previous studies we found that the embryonic stem cell marker 
NANOG is over-expressed in hASCs and its silencing causes downregulation of the OCT4 and SOX2 genes. As assessed by real-time RT-PCR, we confirm that NANOG mRNA in freshly hASC is highly expressed and NANOG knockdown induces OCT4 and SOX2 downregulation, indicating loss of pluripotency in hASCs (data shown in Supplementary Materials).
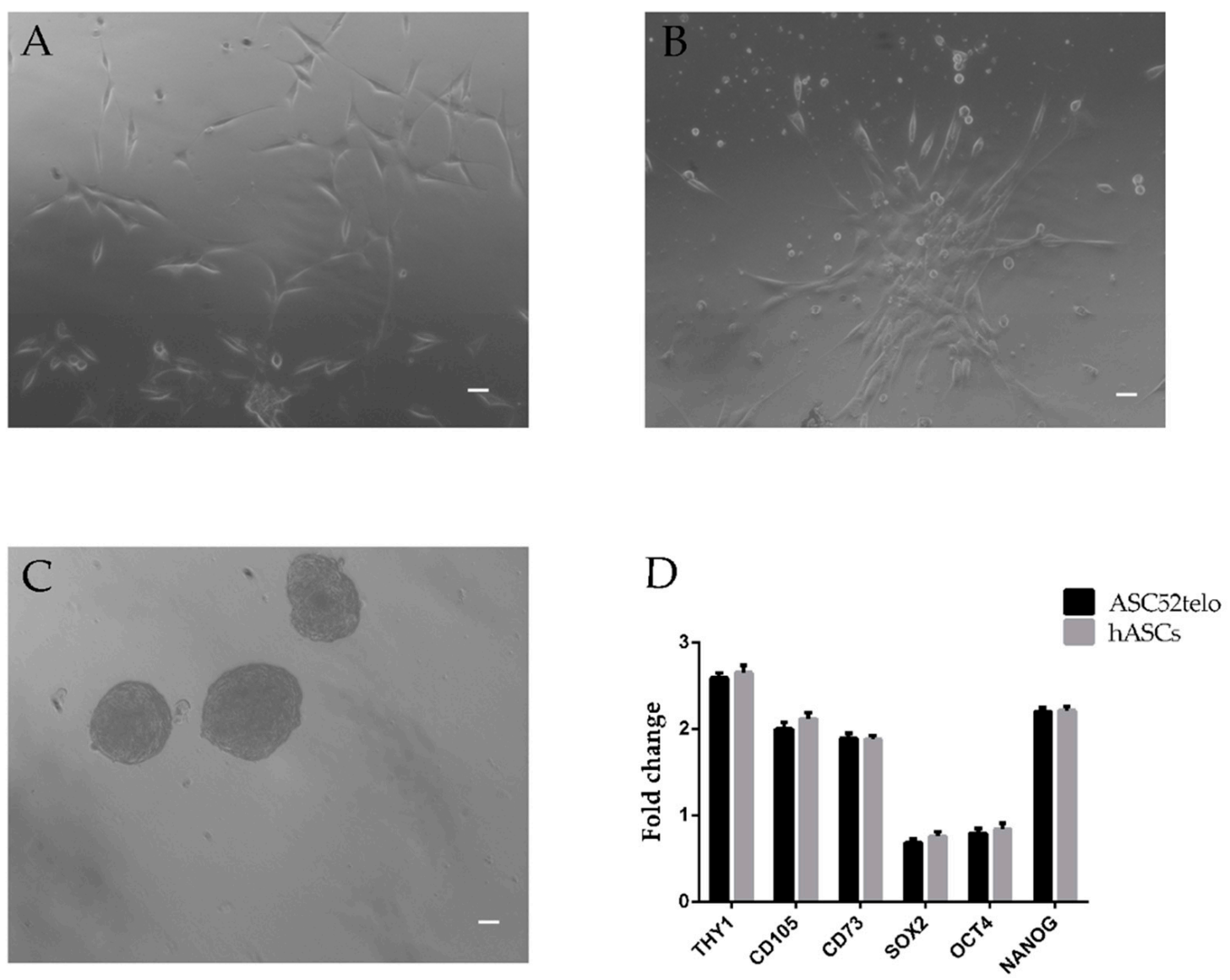

Figure 1. hASC morphology on day 5 (A) of expansion culture observed under light microscopy (10×) with phase contrast with a Nikon DS-FI1 CCD camera. Scale bar $=10 \mu \mathrm{m}$. (B) Shows the formation of hASC colonies when cultured in adhesion, Scale bar $=10 \mu \mathrm{m}$, while $(\mathbf{C})$ shows spheres which formed when hASCs were cultured in low adhesion culture conditions, Scale bar $=10 \mu \mathrm{m}$. A representative experiment referring to 23 samples studied. (D) qRT-PCR analysis in freshly isolated hASCs at passage 4 of THY1, CD73, CD105, OCT4, SOX2 and NANOG gene expression compared to ASC52telo, hTERT immortalized adipose-derived mesenchymal stem cells. Data shown are relative to an endogenous control (beta-Actin). Relative expression levels were assessed using the $2^{-\Delta \Delta C t}$ method. Values are shown as the mean \pm SE. Data are representative of three independent experiments. 

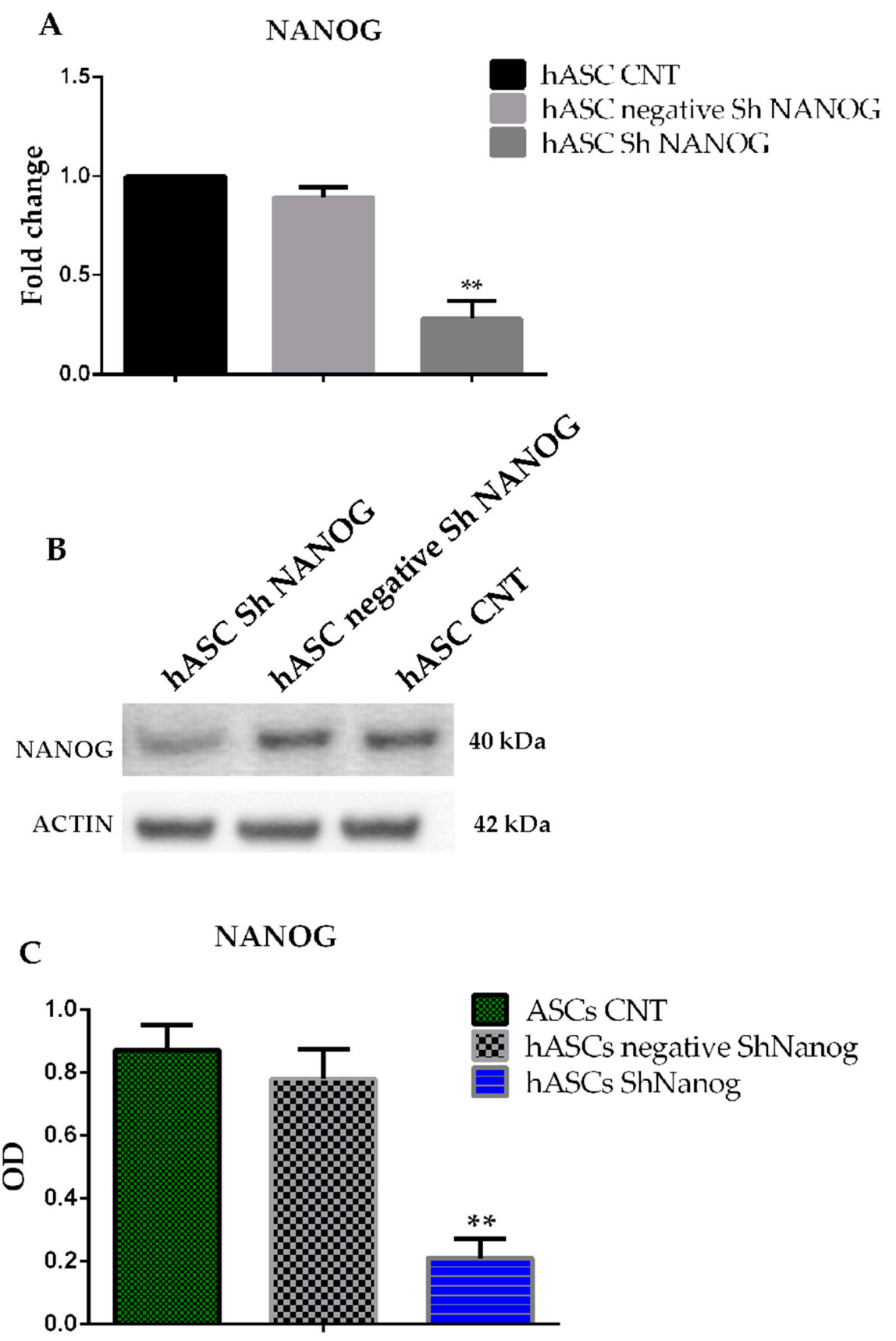

Figure 2. Freshly isolated hASCs at passage 4 (hASCs CNT), hASCs lentivirally transducted with scrambled (negative) or shRNA against NANOG. (A) qRT-PCR analysis for NANOG gene expression after 10-day antibiotic selection. Relative expression levels were assessed using the $2-\Delta \Delta C \mathrm{t}$ method. Data are representative of three independent experiments with the fold change compared to expression levels in a commercial human adipose stem cell line (ASC52telo, hTERT immortalized adipose-derived mesenchymal stem cells). Values are shown as the mean $\pm \mathrm{SE},{ }^{* *} p<0.01$. (B,C) Western blot analysis for protein expression of NANOG after 15 days of antibiotic selection. Values are shown as mean \pm SE, ** $p<0.01$.

\subsection{Analysis of PREF1}

In order better to understand the differentiation potential of hASCs biology, we studied the effect of NANOG shRNA knockdown on DLK1/PREF1 gene expression. We found that PREF1 mRNA was downregulated $(0.8 \pm 0.03$ versus $0.3 \pm 0.06, p<0.01)$ in hASCs with NANOG downregulated (Figure 3$)$. 


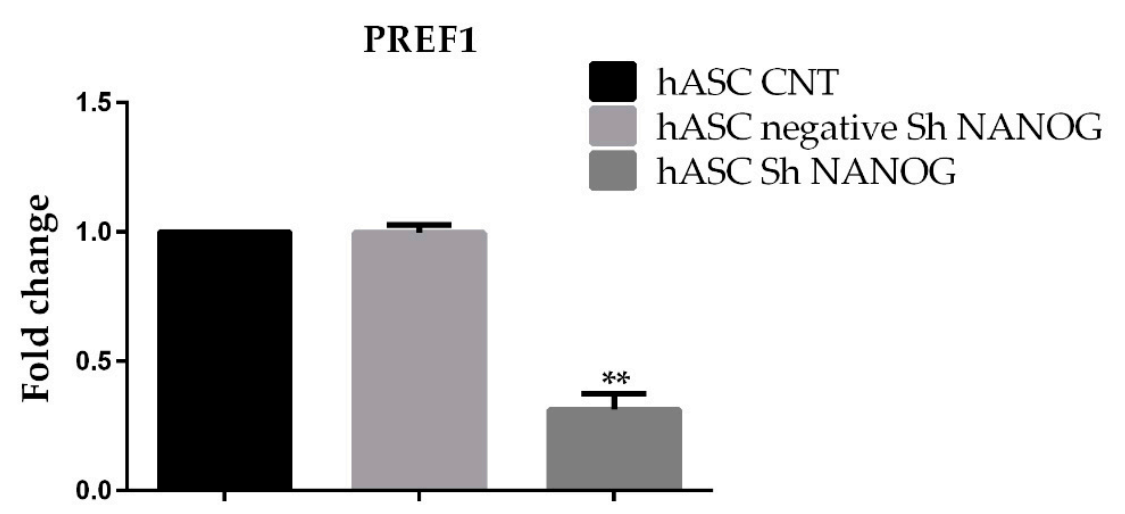

Figure 3. qRT-PCR analysis of PREF1 gene expression in freshly isolated hASCs at passage 4 (hASCs CNT), hASCs lentivirally transducted with scrambled (negative) and shRNA against NANOG (hASCs sh NANOG). PREF1 was significantly downregulated in hASCs sh NANOG. Relative expression levels were assessed using the $2^{-\Delta \Delta C t}$ method. Data are representative of three independent experiments with the fold change compared to expression levels in a commercial human adipose stem cell line (ASC52telo, hTERT immortalized adipose-derived mesenchymal stem cell). The experiment was repeated at least three times ${ }^{* *} p<0.01$.

\subsection{Downregulation of NANOG Inhibited Cell Proliferation, Increased Population Doubling Time and Reduced} Differentiation Potential

The effects of NANOG knockdown on ASCs proliferation were assessed using trypan blue assay and Colony Forming Unit ability. Cell proliferation analysis shows that the hASCs transducted with shRNA against NANOG grew significantly more slowly than wild-type cells and control cells, and after three days, this difference became more evident (Figure 4A). Downregulation of NANOG reduces the number of the colonies. As shown in Figure 4B, the clonogenicity of ASCs transfected with shRNA lentivirus NANOG decreased, according to the number of cell colonies. The colony formation rate of NANOG shRNA-infected cells was $8.5 \pm 3.6 \%$, lower than that of freshly isolated hASCs at passage 4 (hASCs CNT) and scrambled sh NANOG tranfected cells (hASCs negative sh NANOG) ${ }^{* *} p<0.01$ ) and the results show an increase in the population doubling time (Figure 4C). ASCs with NANOG knockdown also show a decrease in adipogenic differentiation potential (Figure 4D). Taken together, hASCs transducted with shRNA against NANOG decrease in proliferation capacity and differentiation potential, indicating that $N A N O G$ has a role in regulation of stem cell properties.

2.5. Knockdown of NANOG Inhibited the Expression of CCND1/Cycline D1, Enhanced the Expression of p21 and $p 27$ and Induced Cell Cycle Block in the G0/G1 Phase

We evaluated the effects of NANOG knockdown on the growth capability of ASCs, and our results confirm that in NANOG knockdown the percentage of cells in G0/G1 phase increased from $48 \pm 3.2 \%$ for freshly isolated hASCs at passage 4 (hASCs CNT) and $52 \pm 1.8 \%$ for hASCs scrambled shRNA to $70.5 \pm 1.2 \%$ (NANOG knockdown group), while the percentage of $S$ phase cells decreased from $45 \pm 2.3 \%$ for freshly isolated hASCs at passage 4 and $48 \%$ for scrambled (negative shRNA NANOG) to $20 \%$ (NANOG knockdown group). (Figure 5A) These results indicated that downregulation of NANOG expression arrested hASCs in the G0/G1 phase. Consistent with these observations, downregulation of NANOG induced a reduction of the proliferation rate as indicated by the proliferation index (P.I.) $0.52 \pm 0.06 \%$ in hASCs negative for shRNA against NANOG versus P.I $0.23 \pm 0.001$ in hASCs transfected with shRNA against NANOG. To investigate the G0/G1 phase arrest we evaluated the principal genes involved in cell proliferation, p27/CDKN1B (Cyclin-dependent kinase inhibitor 1B) p21/CDKN1A (Cyclin-dependent kinase inhibitor 1A) and CYCLINE D1 by qRT-PCR. Interestingly, as shown in Figure 5, knockdown of NANOG in hASCs increased expression of the CDKN1B and CDKN1A (1.8 \pm 0.08 -fc and 3.8 $\pm 0.05-\mathrm{fc}$, respectively, $\left.{ }^{* *} p<0.01\right)$ mRNA level when compared to the 
negative sh NANOG and freshly isolated hASCs at passage 4 (hASCs CNT) $(0.85 \pm 0.05)$. Significant downexpression of CYCLINE D1 in mRNA levels was detected $\left(0.63 \pm 0.09-\mathrm{fc},{ }^{* *} p<0.01\right)$.

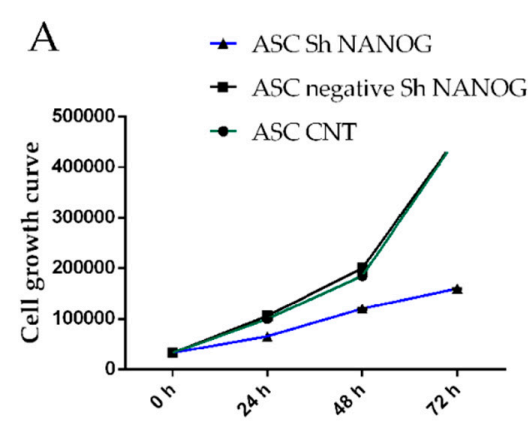

C

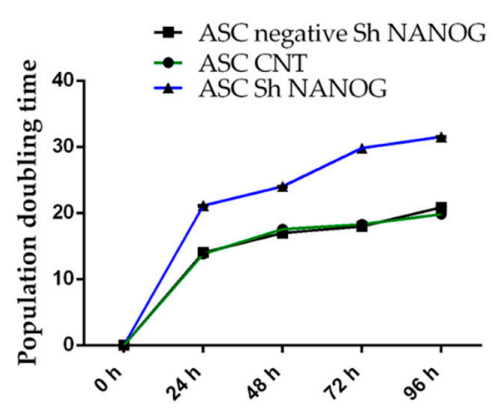

B

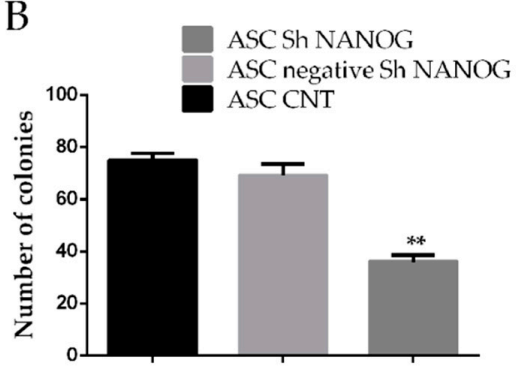

$\mathrm{D}$

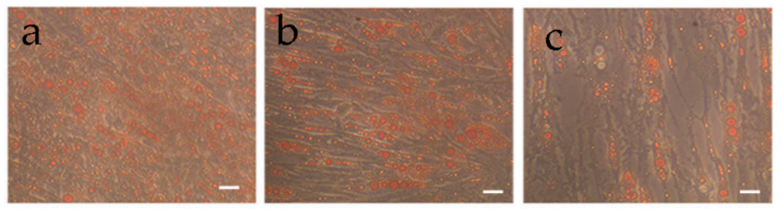

Figure 4. Time-dependent effects of downregulation of NANOG on hASCs growth, colony formation ability, population doubling time and differentiation potential. (A) Shows the cell growth rate over a 72-h period in freshly isolated hASCs at passage 4 (ASC CNT), hASCs lentivirally transduced with scrambled (negative NANOG) or shRNA against NANOG using the trypan blue viability assay. (B) The clone numbers (more than 50 cells) of the freshly isolated hASCs at passage 4 (ASC CNT) and hASCs negative Sh NANOG were much higher than that of the hASCs sh NANOG ( $75 \pm 5$ versus $40 \pm 5$ ). (C) The population doubling time analysis. (D) Shows the differentiation potential into adipogenic lineage: (a) hASCs at passage 4 differentiated into adipocytes, (b) hASCs scrambled sh NANOG tranfected cells (hASCs negative sh NANOG) differentiated into adipocytes and (c) hASCs lentivirally transducted with shRNA against NANOG differentiated into adipocytes. Cultures were observed under light microscopy $(10 \times)$ with phase contrast with a Nikon DS-FI1 CCD camera. scale bar $=10 \mu \mathrm{m}$ (Values are mean \pm SD, $* * p<0.01)$. A representative photograph of the colonies formed 14 days after lentivirus transfection, stained with crystal violet, is shown in Supplementary Materials.

\subsection{Knockdown of NANOG Regulates DNMT1}

To clarify the role of p53 in regulation of $p 21$ and $p 27$ gene expression in hASCs with NANOG downregulated we evaluated p53 mRNA expression. Our findings confirmed that freshly isolated hASCs at passage 4 expressed $p 53$ and NANOG downregulation induced a significant change in $p 53$ $\left(0.5 \pm 0.08-\mathrm{fc}^{*} p<0.05\right)$ gene expression, as shown in Figure 6A. DNA methylation seems to play an essential role in regulating ESC differentiation and embryo development and DNMT-mediated CpG methylation has been demonstrated to play a distinct role in cell cycle regulation. To investigate the possible involvement of DNMTs in cell cycle arrest we evaluated expression of the DNMT1 protein in hASCs lentivirally transduced with shRNA targeting NANOG. We found that DNMT1 protein levels were dramatically reduced in hASCs with NANOG knockdown compared to freshly isolated hASCs at passage 4 and to hASCs scrambled (optical density $(\mathrm{OD})=0.2 \pm 0.03$ versus $0.4 \pm 0.06$ ). (Figure $6 \mathrm{~B}, \mathrm{C}$ ). 

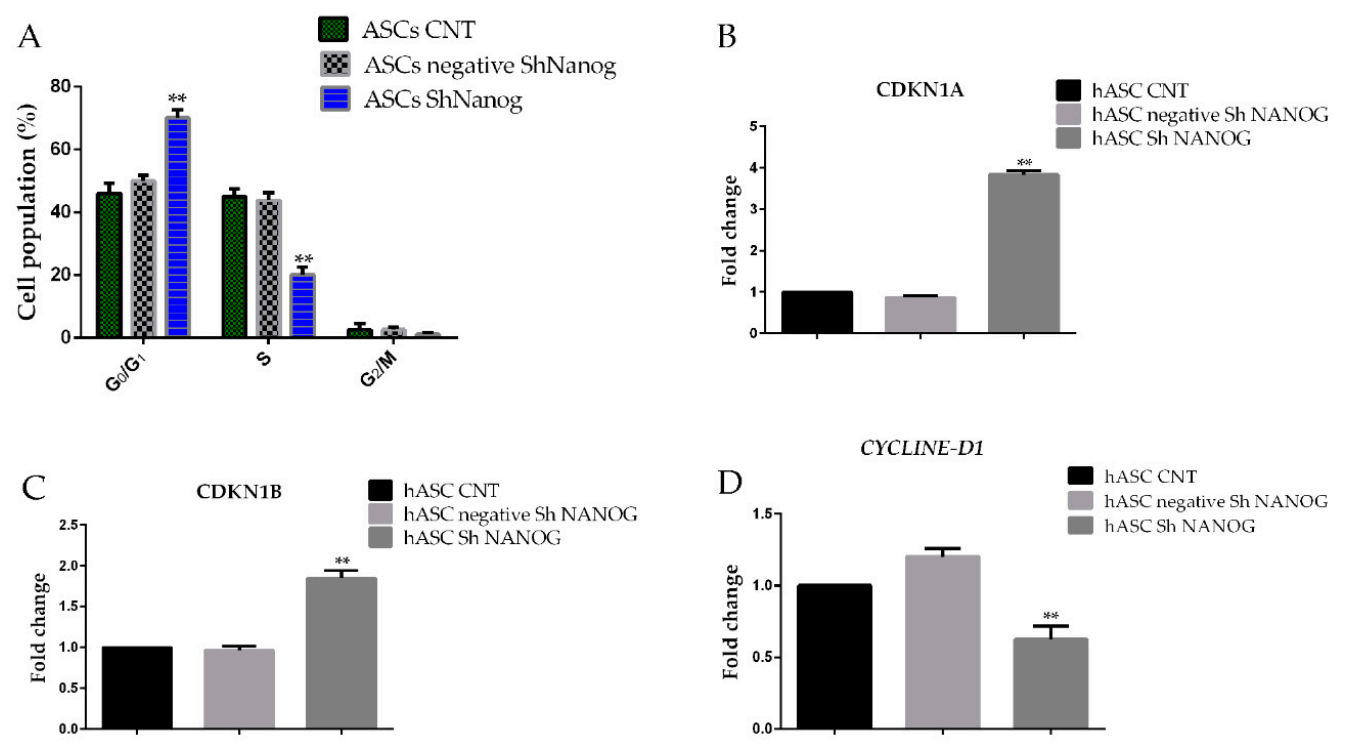

Figure 5. Changes in hASCs cell cycle distribution as a result of NANOG downregulation. (A) Shows cell cycle distribution of hASCs according to Nicoletti's protocol. (B) and (C) Real-time PCR analyses showed that expression of the cell cycle protein CDKN1A and CDKN1B were significantly increased in hASCs with NANOG downregulated while the expression of CYCLINE D1 was downregulated (D). Flow cytometry analyses showed that downregulation of NANOG induced a decrease in the proportion of $\mathrm{G} 2 / \mathrm{M}+\mathrm{S}$ phase cells in hASCs sh NANOG as compared to freshly isolated hASCs at passage 4 (hASCs CNT). All experiments were repeated at least three times. (Values are mean $\pm \mathrm{SD},{ }^{* *} p<0.01$ ).

A
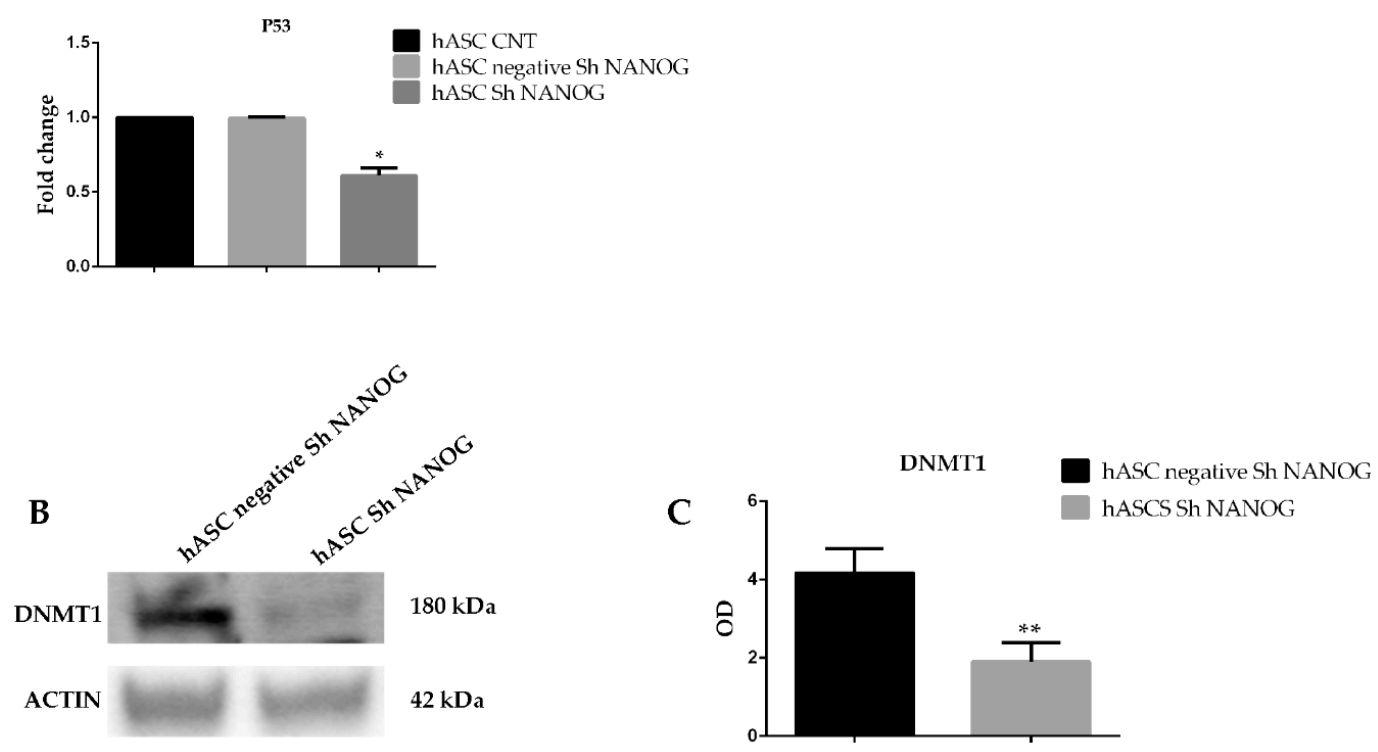

Figure 6. (A) qRT-PCR of $p 53$ gene expression in in freshly isolated hASCs at passage 4 (hASCs CNT), hASCs lentivirally transducted with scrambled (negative) and shRNA against NANOG (hASCs sh NANOG). p53 gene expression was significantly downregulated following inhibition of NANOG in hASCs. Relative expression levels were assessed using the $2^{-\Delta \Delta C t}$ method. Data are representative of three independent experiments with the fold change compared to expression levels in a commercial human adipose stem cell line (ASC52telo, hTERT immortalized adipose-derived mesenchymal stem cell). The experiment was repeated at least three times ${ }^{*} p<0.05$. (B) Representative western blot analysis for expression of DNMT1. Densitometric analysis of the Western blot depicted in (C). The histograms are the results of three independent experiments. The values are shown as the mean $\pm \mathrm{SE}$, ** $p<0.01$. OD, optical density (** $p<0.01)$. 


\section{Discussion}

The properties of stem cells are indicated as the ability to proliferate and to preserve an undifferentiated state and the capacity to differentiate toward multiple cell lineages. It is well known that SOX2 OCT4 and NANOG are the core of "pluripotency" machinery. Our previous study suggested that NANOG is an essential transcriptional regulator of genes involved in maintaining undifferentiated pluripotent state of hASCs, but few data exist to conclude that the hASCs fate and differentiation potential are regulated by this protein. In this study hASCs were isolated from biopsies of subcutaneous adipose tissue undergoing elective open-abdominal surgery and were characterized for stem cell markers CD90, CD105 and CD73, and then, cells were transducted with short hairpin Lentivirus system targeting $N A N O G$, which resulted in significant downregulation of NANOG gene and protein expression. Inhibition of NANOG led to a significant downregulation of OCT4 and $S O X 2$, as previously demonstrated [13], and we were able to detect a decrease in expression of the adipose stem cell marker DLK/PREF1. Knockdown of NANOG in hASCs reduced cell proliferation rate and differentiation potential, inducing cell cycle arrest in G0/G1. p21/CDKN1A and p27/CDKN1B are proteins of the Cip/Kip family that act as key cell cycle regulators by inhibiting CDK $[27,28]$. To understand the roles of NANOG in cell cycle arrest, we examined $p 21$ and $p 27$ gene expression. We demonstrate that downregulation of NANOG in hASCs induced an increase of $p 27$ and $p 21$ gene expression. Recent studies have identified CDK1 as an essential in vivo target of $p 27$ [29] and our in vitro study confirms that CCND1 gene expression is reduced. It is well known that $p 21$ and p27 expression is regulated largely at the transcriptional level by a p53-dependent mechanism [30], which suggests that NANOG may regulate expression of these "player" genes through the $p 53$ tumor suppressor gene. Our experiments confirmed that NANOG downregulation induced a reduction of $p 53$ gene expression, suggesting that NANOG can regulate $p 21$ and $p 27$ activity through $p 53$ activity. Furthermore, it is well known that $p 21$ and $p 27$ are regulated by methylation [31,32] and DNA methylation regulate the expression of appropriate genes in ESCs [31]. Previous experiments have shown in methylation-deficient mouse embryos (DNMT1 ${ }^{-/-}, \mathrm{DNMT}_{3} a^{-/-}$and $\mathrm{DNMT3b^{-/- }}$ ) that the restoring of DNA methylation is essential for development [31-33]. One of the most important DNA methyltransferases is DNMT1, which is responsible for maintaining methylation status during DNA replication, and a recent study demonstrated that NANOG directly binds to the promoter of DNMT1 in ESCs and enhances its expression [26]. To determine whether NANOG-mediated maintenance of stem cell properties in hASC came about through regulation of DNMT1, we evaluated DNMT1 protein expression. The results confirm that NANOG downregulation induced a reduction of DNMT1 protein expression. In conclusion, our results demonstrate that inhibition of NANOG not only decreased hASCs growth and induced the arrest of the cell cycle, but also induced a reduction of differentiation ability. Moreover, our results confirm that the stem cell transcription factor NANOG regulated cell cycle progression probably via $p 53$, which directly controls $p 21$ and $p 27$, key regulator factors of cell cycle machinery. Furthermore, through DNMT1 NANOG can methylate PREF1, which induces differentiation and can modulate the methylation status of $p 21$ and $p 27$ as previously described. All these data suggest that NANOG is essential for maintaining hASCs properties.

\section{Materials and Methods}

\subsection{Cell Culture and Cell Infection with Lentiviral Particles}

Subcutaneous (SAT) adipose tissue biopsies were obtained from 23 consenting patients (10 men, 13 women; age $40 \pm 10$; BMI range between $28 \pm 3$ ) undergoing elective open-abdominal and laparoscopy surgery. The protocol was approved by the Independent Ethical Committee (no. 08/2018; 27 August 2018) at the P. Giaccone Azienda Ospedaliera Universitaria Policlinico, Palermo, Italy. All patients gave their written informed consent. Adipose tissue was processed as previously described [13]. Primary cells were used at passage 4 for all experiments. The commercial primary cell line immortalized with a human telomerase reverse transcriptase (ASC52telo, hTERT) (ATCC@SCRC-4000 ${ }^{\mathrm{TM}}$, American 
Type Culture Collection, Manassas, VA, USA) was cultured in Mesenchymal Stem Cell Basal Medium (ATCC PCS-500-030).supplemented with Mesenchymal Stem Cell Growth Kit (ATCC PCS-500-040, LGC Standards, Milan, Italy). For lentivirus infection, $2 \times 10^{4} \mathrm{hASC}$ sere seeded in six-well plates, and infected with lentivirus (sc-43958-V, Santa Cruz Biotechnology, DBA, Milan, Italy with a the multiplicity of infection (MOI) 10 in the presence of $8 \mu \mathrm{g} / \mathrm{mL}$ polybrene (sc-134220, Santa Cruz Biotechnology). At $24 \mathrm{~h}$ after infection, the media were removed and replaced with fresh growth media. After $48 \mathrm{~h}$ the media were replaced with fresh growth media containing puromycin $(1 \mu \mathrm{g} / \mathrm{mL}$, cod. P9620, Sigma Aldrich, Milan, Italy) to select for infected cells. All experiments were performed 10-15 days after puromycin selection.

\subsection{RNA Isolation and Quantitative RT-PCR}

RNA was isolated in columns from subconfluent cultures of hASC by RNeasy kit (Qiagen, Hamburg, Germany) as previously described [13]. Gene expression was normalized for the housekeeping gene beta-actin (Invitrogen, Milan, Italy). Amplification of specific transcripts was confirmed by melting curve profiles at the end of each PCR. PCR primers NANOG (QT01844808), OCT3/4 (QT00210840), SOX2 (QT00237601), THY1 (QT00023569), CD105 (QT00013335), CD73 (QT00027279), p53 (QT00060235), CCDN1 (QT00495285), CDKN1A (QT00062090), CDKN1B (QT00998445) were purchased from Qiagen (QuantiTect $®$ Primer Assays, Qiagen, Hamburg, Germany), $\beta$-Actin (FORWARD: 5'-GGACTT CGA GCA AGA GAT GG-3' REVERSE: 5'-AGC ACT GTG TTG GCG TAC AG-3') was purchased from Invitrogen. All reactions were performed using the Quantitect SYBR Green PCR Kit (cod. 204243, Qiagen) on the RotorGene Q Instrument (Qiagen) as previously described. Gene expression of primary cells was compared with a commercial primary cell line immortalized with a human telomerase reverse transcriptase (ASC52telo, hTERT) ATCC®SCRC-4000 ${ }^{\mathrm{TM}}$, American Type Culture Collection, Manassas, VA, USA), as positive cell controls. Relative expression levels for each gene were assessed using the $2^{-\Delta \Delta C t}$ method. The results were represented as histograms with GraphPad Prism 6 Software (GraphPad Software, Inc., La Jolla, CA, USA). qRT-PCR analyses for the stem gene were also performed after lentivirus infection experiments.

\subsection{Western Blot Analysis}

Proteins were extracted from adherent cultured cells and separated as previously described $[13,34,35]$. The antibodies used are as described in Table 1 . The secondary antibody was goat anti-mouse IgG-HRP (Amersham, GE Healthcare Europe GmbH, Milan, Italy). Antigen-antibody complexes were visualized using the ECL prime (Amersham, GE Healthcare Europe GmbH, Milan, Italy) on a CCD camera (Chemidoc, Bio-Rad, Milan, Italy). Western blot bands were quantified with ImageJ 1.48 software (National Institutes of Health, Bethesda, MD, USA) and the results were represented as histograms with GraphPad Prism 6 Software (GraphPad Software, Inc., La Jolla, CA, USA).

Table 1. Primary antibodies used.

\begin{tabular}{cccc}
\hline Primary Antibody/Localization Marker & Code Number & Diluition & Incubation \\
\hline NANOG, nuclear and cytoplasmatic & sc-293121, Santa Cruz Biotechnology & $1: 500$ & $\mathrm{o} / \mathrm{n}, 4^{\circ} \mathrm{C}$ \\
\hline DNMT1, nuclear & sc-271729, Santa Cruz Biotechnology & $1: 500$ & $\mathrm{o} / \mathrm{n}, 4^{\circ} \mathrm{C}$ \\
\hline B-Actin clone AC-74 & A5316, Sigma Aldrich & $1: 10,000$ & $\mathrm{o} / \mathrm{n}, 4^{\circ} \mathrm{C}$ \\
\hline
\end{tabular}

\subsection{Analysis of Cell Cycle Status of MSCs with Lentivirus}

Single-cell suspensions of control samples and transfected samples were obtained and seeded at a density of $2 \times 10^{3}$ cells $/ \mathrm{cm}^{2}$ (passage 3), and the DNA content was assessed according to Nicoletti's protocol [36] as previously described [13]. Data were acquired with CellQuest Pro software 
(Becton Dickinson, Milan, Italy) and the percentages of G1, S and G2 phase cells were calculated with the MODFIT-LT 5.0 software program (Verity Software House Inc., Topsham, ME, USA).

\subsection{Colony-Forming Assay}

Single-cell suspension ASCs cell lines were seeded in a six-well culture in DMEM/Ham's F12 1:1 supplemented with $100 \mathrm{unit} / \mathrm{mL}$ penicillin, $0.1 \mathrm{mg} / \mathrm{mL}$ streptomycin and $10 \%$ fetal calf serum (FCS) at a density of 300 cells/well and cultured at $37{ }^{\circ} \mathrm{C}$ in $5 \% \mathrm{CO}_{2}$. After 14 days, the cells were fixed in $4 \%$ paraformaldehyde (Sigma Aldrich) and stained with $0.1 \%$ crystal violet (Sigma Aldrich). Only the cell groups containing more than 50 cells were considered as colonies. Numbers of colonies were quantified with Image 1.48 software (National Institutes of Health, Bethesda, MD, USA).

\subsection{Population Doubling and Cell Proliferation Curve}

Cell proliferation curve and population doubling time were assessed as previously described [35]. The doubling time (DT) was calculated in accordance with the literature data (http://www.doublingtime.com/compute.php). Three sets of experiments for each sample were used for calculations.

\section{Conclusions}

We examined whether NANOG contributes to maintaining cells in an undifferentiated, pluripotent state by activating certain key genes and by silencing others. For this purpose, hASCs were transfected with a lentivirus with shRNA targeting NANOG and our results suggest that NANOG plays a key role in the hASCs proliferation rate by increasing the expression of $p 21$ and $p 27$ and by modulating PREF1. In conclusion, we confirm that NANOG is an important player in the complex transcription network that regulates pluripotency. We hypothesized that $p 21, p 27$ and PREF1 may be regulated by DNMT1, a promotor that is directly bound by NANOG, as demonstrated by Tsai et al. [26]. Further experiments are needed to establish the pathway to explain the involvement of NANOG in the control of cell cycle progression.

Supplementary Materials: Supplementary Materials can be found at http:/www.mdpi.com/1422-0067/20/10/ 2580/s1.

Author Contributions: M.P. was responsible for the conception of the work and the design, collection and assembly of data, data analysis and interpretation and manuscript writing. G.P. (Giuseppe Pizzolanti) was responsible for acquisition of data and revision of the manuscript. S.M. and G.P. (Gianni Pantuso) were responsible for provision of biopsies. M.P., G.P. (Giuseppe Pizzolanti), L.T. and A.C. were responsible for data analysis and interpretation and drafting of the manuscript. C.G. was responsible for the conception of the work and the design, data analysis and interpretation, manuscript writing and final approval of the manuscript, manuscript drafting, and critically revision for important intellectual content and financial support. All authors agree to be accountable for all aspects of the work in ensuring that questions related to the accuracy or integrity of any part of the work are appropriately investigated and resolved. All authors read and approved the final manuscript.

Funding: FFR2018 to C.G.

Acknowledgments: This work is dedicated to our laboratory staff.

Conflicts of Interest: The authors declare no conflict of interest.

\section{Abbreviations}

MSC

hASC

p21/CDKN1A

p27/CDKN1B
Mesenchymal Stem Cell

human Adipose Stem Cell

Cyclin-dependent kinase inhibitor $1 \mathrm{~A}$

Cyclin-dependent kinase inhibitor 1B 


\section{References}

1. Boyer, L.A.; Lee, T.I.; Cole, M.F.; Johnstone, S.E.; Levine, S.S.; Zucker, J.P.; Guenther, M.G.; Kumar, R.M.; Murray, H.L.; Jenner, R.G.; et al. Core transcriptional regulatory circuitry in human embryonic stem cells. Cell 2005, 122, 947-956. [CrossRef] [PubMed]

2. Pan, G.; Thomson, J.A. Nanog and transcriptional networks in embryonic stem cell pluripotency. Cell Res. 2007, 17, 42-49. [CrossRef]

3. Kim, J.; Chu, J.; Shen, X.; Wang, J.; Orkin, S.H. An Extended Transcriptional Network for Pluripotency of Embryonic Stem Cells. Cell 2008, 132, 1049-1061. [CrossRef] [PubMed]

4. Darr, H. Overexpression of NANOG in human ES cells enables feeder-free growth while inducing primitive ectoderm features. Development 2006, 133, 1193-1201. [CrossRef]

5. Hough, S.R.; Clements, I.; Welch, P.J.; Wiederholt, K.A. Differentiation of Mouse Embryonic Stem Cells after RNA Interference-Mediated Silencing of OCT4 and Nanog. Stem Cells 2006, 24, 1467-1475. [CrossRef] [PubMed]

6. Hyslop, L.; Stojkovic, M.; Armstrong, L.; Walter, T.; Stojkovic, P.; Przyborski, S.; Herbert, M.; Murdoch, A.; Strachan, T.; Lako, M. Downregulation of NANOG Induces Differentiation of Human Embryonic Stem Cells to Extraembryonic Lineages. Stem Cells 2005, 23, 1035-1043. [CrossRef] [PubMed]

7. Wang, Z.; Oron, E.; Nelson, B.; Razis, S.; Ivanova, N. Distinct lineage specification roles for NANOG, OCT4, and SOX2 in human embryonic stem cells. Cell Stem Cell 2012, 10, 440-454. [CrossRef] [PubMed]

8. Li, A.I.; Hokugo, A.; Jarrahy, R.; Zuk, P.A. Human adipose tissue as a source of multipotent stem cells. In Stem Cells in Aesthetic Procedures: Art, Science, and Clinical Techniques; Springer-Verlag GmbH: Heidelberg, Germany, 2014.

9. Zuk, P.A.; Zhu, M.; Mizuno, H.; Huang, J.; Futrell, J.W.; Katz, A.J.; Benhaim, P.; Lorenz, H.P.; Hedrick, M.H. Multilineage cells from human adipose tissue: Implications for cell-based therapies. Tissue Eng. 2001, 7, 211-228.

10. Gronthos, S.; Franklin, D.M.; Leddy, H.A.; Robey, P.G.; Storms, R.W.; Gimble, J.M. Surface protein characterization of human adipose tissue-derived stromal cells. J. Cell. Physiol. 2001, 189, 54-63. [CrossRef]

11. Dominici, M.; Le Blanc, K.; Mueller, I.; Slaper-Cortenbach, I.; Marini, F.; Krause, D.; Deans, R.; Keating, A.; Prockop, D.; Horwitz, E. Minimal criteria for defining multipotent mesenchymal stromal cells. The International Society for Cellular Therapy position statement. Cytotherapy 2006, 8, 315-317. [CrossRef]

12. De Francesco, F.; Tirino, V.; Desiderio, V.; Ferraro, G.; D’Andrea, F.; Giuliano, M.; Libondi, G.; Pirozzi, G.; De Rosa, A.; Papaccio, G. Human CD34+/CD90+ ASCs are capable of growing as sphere clusters, producing high levels of VEGF and forming capillaries. PLoS ONE 2009, 4, e6537. [CrossRef] [PubMed]

13. Pitrone, M.; Pizzolanti, G.; Tomasello, L.; Coppola, A.; Morini, L.; Pantuso, G.; Ficarella, R.; Guarnotta, V.; Perrini, S.; Giorgino, F.; et al. NANOG plays a hierarchical role in the transcription network regulating the pluripotency and plasticity of adipose tissue-derived stem cells. Int. J. Mol. Sci. 2017, 18, 1107. [CrossRef]

14. Potdar, P.D.; Sutar, J.P. Establishment and molecular characterization of mesenchymal stem cell lines derived from human visceral \& subcutaneous adipose tissues. J. Stem Cells Regen. Med. 2010, 6, 26-35. [PubMed]

15. Langroudi, L.; Forouzandeh, M.; Soleimani, M.; Atashi, A.; Golestaneh, A.F. Induction of differentiation by down-regulation of Nanog and Rex-1 in cord blood derived unrestricted somatic stem cells. Mol. Biol. Rep. 2013, 40, 4429-4437. [CrossRef] [PubMed]

16. Perrini, S.; Ficarella, R.; Picardi, E.; Cignarelli, A.; Barbaro, M.; Nigro, P.; Peschechera, A.; Palumbo, O.; Carella, M.; De Fazio, M.; et al. Differences in Gene Expression and Cytokine Release Profiles Highlight the Heterogeneity of Distinct Subsets of Adipose Tissue-Derived Stem Cells in the Subcutaneous and Visceral Adipose Tissue in Humans. PLoS ONE 2013, 8, e57892. [CrossRef] [PubMed]

17. Lengner, C.J.; Camargo, F.D.; Hochedlinger, K.; Welstead, G.G.; Zaidi, S.; Gokhale, S.; Scholer, H.R.; Tomilin, A.; Jaenisch, R. Oct4 Expression Is Not Required for Mouse Somatic Stem Cell Self-Renewal. Cell Stem Cell 2007, 1, 403-415. [CrossRef]

18. Rodeheffer, M.S.; Birsoy, K.; Friedman, J.M. Identification of White Adipocyte Progenitor Cells In Vivo. Cell 2008, 135, 240-249. [CrossRef]

19. Smas, C.M.; Sul, H.S. Pref-1, a protein containing EGF-like repeats, inhibits adipocyte differentiation. Cell 1993, 73, 725-734. [CrossRef] 
20. Tang, W.; Zeve, D.; Suh, J.M.; Bosnakovski, D.; Kyba, M.; Hammer, R.E.; Tallquist, M.D.; Graff, J.M. White fat progenitor cells reside in the adipose vasculature. Science 2008, 322, 583-586. [CrossRef]

21. Tseng, Y.H.; Butte, A.J.; Kokkotou, E.; Yechoor, V.K.; Taniguchi, C.M.; Kriauciunas, K.M.; Cypess, A.M.; Niinobe, M.; Yoshikawa, K.; Patti, M.E.; et al. Prediction of preadipocyte differentiation by gene expression reveals role of insulin receptor substrates and necdin. Nat. Cell Biol. 2005, 7, 601-611. [CrossRef] [PubMed]

22. Wang, Y.; Hudak, C.; Sul, H.S. Role of preadipocyte factor 1 in adipocyte differentiation. Clin. Lipidol. 2010, 5 , 109-115. [CrossRef]

23. Wang, Y.; Kim, K.-A.; Kim, J.-H.; Sul, H.S. Pref-1, a Preadipocyte Secreted Factor That Inhibits Adipogenesis. J. Nutr. 2018, 136, 2953-2956. [CrossRef] [PubMed]

24. Mitterberger, M.C.; Lechner, S.; Mattesich, M.; Kaiser, A.; Probst, D.; Wenger, N.; Pierer, G.; Zwerschke, W. DLK1(PREF1) is a negative regulator of adipogenesis in CD105+/CD90+/CD34+/CD31-/FABP4adipose-derived stromal cells from subcutaneous abdominal fat pats of adult women. Stem Cell Res. 2012, 9, 35-48. [CrossRef]

25. Biniszkiewicz, D.; Gribnau, J.; Ramsahoye, B.; Gaudet, F.; Eggan, K.; Humpherys, D.; Mastrangelo, M.-A.; Jun, Z.; Walter, J.; Jaenisch, R. Dnmt1 overexpression causes genomic hypermethylation, loss of imprinting, and embryonic lethality. Mol. Cell. Biol. 2002, 22, 2124-2135. [CrossRef] [PubMed]

26. Tsai, C.C.; Su, P.F.; Huang, Y.F.; Yew, T.L.; Hung, S.C. Oct4 and Nanog Directly Regulate Dnmt1 to Maintain Self-Renewal and Undifferentiated State in Mesenchymal Stem Cells. Mol. Cell 2012, 47, 169-182. [CrossRef]

27. Wade Harper, J.; Adami, G.R.; Wei, N.; Keyomarsi, K.; Elledge, S.J. The p21 Cdk-interacting protein Cip1 is a potent inhibitor of G1 cyclin-dependent kinases. Cell 1993, 75, 805-816. [CrossRef]

28. Xiong, Y.; Hannon, G.J.; Zhang, H.; Casso, D.; Kobayashi, R.; Beach, D. P21 is a universal inhibitor of cyclin kinases. Nature 1993, 366, 701-704. [CrossRef]

29. Aleem, E.; Kiyokawa, H.; Kaldis, P. Cdc2-cyclin E complexes regulate the G1/S phase transition. Nat. Cell Biol. 2005, 7, 831-836. [CrossRef]

30. Gartel, A.L.; Tyner, A.L. Transcriptional regulation of the p21((WAF1/CIP1)) gene. Exp. Cell Res. 1999, 246, 280-289. [CrossRef]

31. Fouse, S.D.; Shen, Y.; Pellegrini, M.; Cole, S.; Meissner, A.; Van Neste, L.; Jaenisch, R.; Fan, G. Promoter CpG Methylation Contributes to ES Cell Gene Regulation in Parallel with Oct4/Nanog, PcG Complex, and Histone H3 K4/K27 Trimethylation. Cell Stem Cell 2008, 2, 160-169. [CrossRef]

32. Lei, H.; Oh, S.P.; Okano, M.; Jüttermann, R.; Goss, K.A.; Jaenisch, R.; Li, E. De novo DNA cytosine methyltransferase activities in mouse embryonic stem cells. Development 1996, 122, 3195-3205. [PubMed]

33. Okano, M.; Bell, D.W.; Haber, D.A.; Li, E. DNA methyltransferases Dnmt3a and Dnmt3b are essential for de novo methylation and mammalian development. Cell 1999, 99, 247-257. [CrossRef]

34. Coppola, A.; Tomasello, L.; Pitrone, M.; Cillino, S.; Richiusa, P.; Pizzolanti, G.; Giordano, C. Human limbal fibroblast-like stem cells induce immune-tolerance in autoreactive T lymphocytes from female patients with Hashimoto's thyroiditis. Stem Cell Res. Ther. 2017, 8, 154. [CrossRef] [PubMed]

35. Tomasello, L.; Mauceri, R.; Coppola, A.; Pitrone, M.; Pizzo, G.; Campisi, G.; Pizzolanti, G.; Giordano, C. Mesenchymal stem cells derived from inflamed dental pulpal and gingival tissue: A potential application for bone formation. Stem Cell Res. Ther. 2017, 8, 179. [CrossRef] [PubMed]

36. Riccardi, C.; Nicoletti, I. Analysis of apoptosis by propidium iodide staining and flow cytometry. Nat. Protoc. 2006, 1, 1458-1461. [CrossRef] [PubMed]

(C) 2019 by the authors. Licensee MDPI, Basel, Switzerland. This article is an open access article distributed under the terms and conditions of the Creative Commons Attribution (CC BY) license (http://creativecommons.org/licenses/by/4.0/). 